\title{
Fusarium mycotoxins in UK straw from the 2008 harvest - Implications for pigs on straw bedding
}

S G Edwards, A H Stewart

Harper Adams University College, Newport, Shropshire, United Kingdom

Email:astewart@harper-adams.ac.uk

Introduction Straw based production systems are common in the UK compared to other countries. The effects of mycotoxins in cereal feed on livestock performance are relatively well documented with pigs being particularly sensitive to mycotoxicosis. The European Commission has recently set guideline limits for fusarium mycotoxins in animal feedstuffs (Anon, 2006). Guidance limits for feedstuffs for young pigs is $900 \mathrm{ppb}$ deoxynivalenol and 100 ppb zearalenone. A previous study in 2006 identified straw as a potential source of fusarium mycotoxins for livestock on straw bedding. The cereal harvest in 2008 was the wettest for many years in the UK. This resulted in severe losses in grain quality and the highest recorded levels of fusarium mycotoxins in UK wheat and barley grain samples. This project was conducted to evaluate the corresponding levels of fusarium mycotoxins in straw from the 2008 harvest.

Material and methods A total of 58 samples of wheat and barley straw were collected from pig farms across England and Scotland. All straw samples were from the 2008 harvest and collected in July 2009. Samples (ca. 500 g) were collected from several points in the interior of a cut bale. Each sample was dried to ca. 12\% moisture content and milled in a hammer mill with a $1 \mathrm{~mm}$ screen. Zearalenone (ZON) and deoxynivalenol (DON) were analysed using Ridascreen DON and Zearalenone ELISA test kits (R-Biopharm Rhone), which based on the modified extraction procedure had limits of quantification for DON and ZON of 75 and 7 parts per billion (ppb) respectively. Mycotoxin data were normalised using a $\log$ transformation and wheat and barley mycotoxin levels compared by unbalanced ANOVA using Genstat software (version 12).

Results The concentrations of fusarium mycotoxins in wheat and barley straw (Table 1) were higher than those found in corresponding wheat and barley grain samples from the same harvest (HGCA, 2009). The concentrations were also higher than those found in a previous study of wheat straw in 2005 (White et al. 2007), particularly for ZON. There was significantly $(\mathrm{P}<0.001)$ higher $\mathrm{DON}$ and $\mathrm{ZON}$ in wheat compared to barley straw. High concentrations of fusarium mycotoxins, however, existed in both barley and wheat samples with both cereals having maximum DON and ZON concentrations of ca. 2000 and 4000 ppb respectively. Five barley samples (17\%) exceeded the ZON guidance limit for piglet feed (100 ppb) and two samples (7\%) exceeded the DON guidance limit for pig feed (900 ppb). Twelve wheat samples (44\%) exceeded the ZON guidance limit for piglet feed (100 ppb) and 13 samples (48\%) exceeded the DON guidance limit for pig feed (900 ppb).

Table 1 Mean DON and ZON concentrations from UK barley and wheat straw and grain from various studies

\begin{tabular}{llllll}
\hline \hline Year & Cereal & Product & DON $(\mathrm{ppb})$ & ZON $(\mathrm{ppb})$ & Reference \\
\hline 2005 & Wheat & Straw & 460 & 23 & White et al. 2007 \\
2008 & Barley & Grain & 32 & 36 & HGCA 2009 \\
2008 & Wheat & Grain & 584 & 121 & HGCA 2009 \\
2008 & Barley & Straw & 166 & 12 & This study \\
2008 & Wheat & Straw & 983 & 499 & This study \\
\hline \hline
\end{tabular}

Conclusions UK cereal straw, in particular wheat straw, can contain high levels of fusarium mycotoxins. There are limited data on the rate of consumption of bedding straw, however one study calculated weaned pigs consumed about 1.6 $\mathrm{kg}$ per day. Based on the levels of fusarium mycotoxins found in straw, then this could be a significant proportion of the mycotoxin load consumed by pigs and contribute to sub-clinical (reduced weight gain) and clinical mycotoxicosis. There have been several cases of ZON mycotoxicosis reported within the pig industry since the 2008 harvest. Zearalenone mimics oestrogen resulting in hyperoestrogenism. Symptoms reported include swollen vulva in newborn piglets, reduced litter numbers and increased numbers of weak and/or deformed piglets at birth. Results from this study would indicate that mycotoxins within bedding straw could contribute to mycotoxicosis. Farmers using a straw bedding system should therefore consider straw as a component of the diet and as such it should be tested as part of any veterinary investigation of mycotoxicosis.

Acknowledgements This project was funded by Alltech (UK) Ltd. We also thank the commercial producers that kindly supplied samples and Terry McArdle (Alltech) and Allan Ward (QMS) who coordinated sampling in England and Scotland respectively.

\section{References}

Anon. 2006. Official Journal of the European Union L229, 7-9

HGCA. 2009. Available online at http://www.hgca.com

White, C.I., Edwards, S.G. and Stewart, A.H. 2007. Proceedings of the British Society of Animal Science, 106 